\title{
Novel Tools to Dissect the Dynamic Regulation of TCR Signaling by the Kinase Csk and the Phosphatase CD45
}

\author{
Ying Xim TAN, ${ }^{1,2,3,5}$ JUlie Zikherman, ${ }^{1,2,3,5}$ AND ARThur Weiss ${ }^{1,2,3,4}$ \\ ${ }^{1}$ Division of Rheumatology, UCSF, San Francisco, California 94143 \\ ${ }^{2}$ Rosalind Russell Medical Research Center for Arthritis, UCSF, San Francisco, California 94143 \\ ${ }^{3}$ Department of Medicine, UCSF, San Francisco, California 94143 \\ ${ }^{4}$ Howard Hughes Medical Institute, UCSF, San Francisco, California 94143 \\ Correspondence: aweiss@medicine.ucsf.edu
}

\begin{abstract}
Although the biochemical events induced by T-cell receptor (TCR) triggering have been well studied, both the mediators and function of basal signaling in $\mathrm{T}$ cells remain poorly understood. Furthermore, the precise mechanisms by which MHCpeptide interaction with the TCR disrupt the basal equilibrium to induce downstream signaling are also unclear. Here we describe novel approaches to understand the basal state of $\mathrm{T}$ cells and the mechanisms of TCR triggering by perturbing regulation of the Src family kinases (SFKs). The SFKs are critical proximal mediators of TCR signaling that are in turn tightly regulated by the tyrosine kinase Csk and the receptor-like tyrosine phosphatase CD45. We have developed a small-molecule analog-sensitive allele of Csk and an allelic series of mice in which expression of CD45 is varied across a broad range. Our studies have unmasked contributions of Csk and CD45 to maintain the basal state of T cells and also suggest that dynamic regulation of Csk may be involved in TCR triggering.
\end{abstract}

The T-cell receptor (TCR) is composed of distinct antigen recognition components (TCR- $\alpha \beta$ chains) and signal transduction components (CD3 and $\zeta$ chains). Upon peptide/MHC ( $\mathrm{pMHC}$ ) recognition, the TCR activates a well-studied signal transduction cascade. The CD4/CD8 coreceptor-associated Src family tyrosine kinase (SFK) Lck is recruited to the TCR where it phosphorylates the immunoreceptor tyrosine based activation motif (ITAM) tyrosines of the CD3 and $\zeta$ chains. This favors recruitment of the Syk family kinase ZAP70, which is, in turn, phosphorylated by Lck (Weiss 1993; Wang et al. 2010). Lck forms a complex with ZAP-70 via binding its SH2 domain to phospho-Y319 (Thome et al. 1995; Straus et al. 1996). This latter event positions Lck in close proximity to ZAP-70 to amplify signaling and also may contribute to the stabilization of the interaction of the CD4/CD8 coreceptors with pMHC complexes (Xu and Littman 1993; Artyomov et al. 2010). Together Lck and ZAP-70 phosphorylate and activate a critical "signalosome" nucleated by the adaptors Lat and SLP-76 (Koretzky et al. 2006). This signalosome recruits a variety of effector proteins such as PLC $\gamma 1$, which in turn activate diverse downstream signaling pathways leading to a variety of biological outcomes including cell activation, proliferation, differentiation, or death depending on cellular context.

Given its critical role in initiating TCR signaling, it is not surprising that Lck is itself tightly regulated by two tyrosine phosphorylation sites (Hermiston et al. 2009). Phosphorylation of the activation loop tyrosine (Y394) is required for full Lck kinase activity, whereas phosphorylation of the carboxy-terminal inhibitory site (Y505) favors adoption of a closed auto-inhibited conformation that stabilizes the inactive conformation of the catalytic domain. This inhibitory tyrosine is in turn reciprocally regulated by the enzymatic activities of the receptorlike tyrosine phosphatase CD45 and the cytoplasmic tyrosine kinase Csk (Fig. 1). Together this phosphatase/ kinase pair impose tight, constitutive control over Lck activity and shape both inducible and tonic signaling tone. Here, we highlight recent work from our laboratory that sheds light on how CD45 and Csk regulate both basal and inducible TCR signaling.

\section{MODELS OF TCR ACTIVATION}

Despite extensive studies of TCR signal transduction, how ligation of the TCR $-\alpha \beta$ chains is coupled to downstream signaling events remains uncertain. Several models that are not mutually exclusive have been proposed. One such model suggests that a conformational change is transmitted structurally from the extracellular TCR- $\alpha \beta$ chains to the CD3 and $\zeta$ chain cytoplasmic ITAMs (Gil et al. 2002; Call and Wucherpfennig 2004; Levin and Weiss 2005). Attempts to validate such mechanisms have yielded conflicting results (La Gruta et al. 2004; Mingueneau et al. 2008). TCR dimerization or oligomerization in the presence of ligand has also been proposed to occur and mediate T-cell activation (Boniface et al. 1998). Another model posits that MHC recruitment of CD4/CD8 coreceptor-associated Lck couples ligand recognition to signal transduction (Weiss 1993; Xu and Littman 1993; Artyomov et al. 2010). However, studies of

\footnotetext{
${ }^{5}$ These authors contributed equally.

Copyright (C) 2013 Cold Spring Harbor Laboratory Press; all rights reserved; doi: 10.1101/sqb.2013.78.020347

Cold Spring Harbor Symposia on Quantitative Biology, Volume LXXVIII
} 


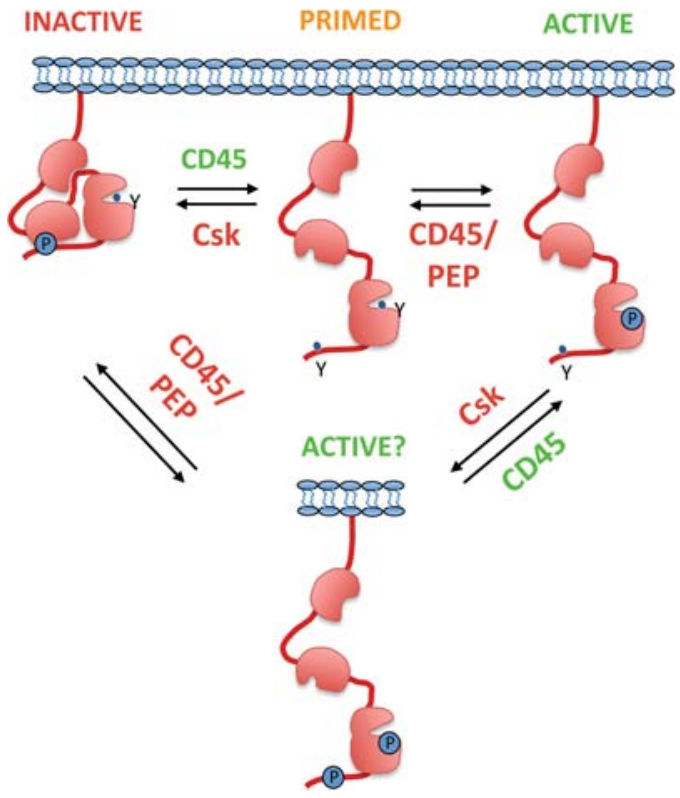

Figure 1. SFK activity is reciprocally regulated by Csk and CD45. Phosphorylation of a carboxy-terminal tail negative regulatory tyrosine of SFKs by Csk facilitates its interaction with its $\mathrm{SH} 2$ domain, resulting in a closed, catalytically inactive conformation. Dephosphorylation of this site by CD45 favors an open conformation. Phosphorylation of the catalytic site tyrosine is required for full kinase activity. CD45 and PEP can negatively regulate SFK activity by dephosphorylating the catalytic site tyrosine.

$\mathrm{T}$ cells lacking coreceptors have shown that coreceptors impose MHC restriction but can be dispensible for ligand-mediated TCR signaling (Locksley et al. 1993; Van Laethem et al. 2007). Over the past decade, there has been growing experimental support for the so-called "kinetic segregation" model of TCR activation, first proposed by Van der Merwe and Davis in 1996 (Davis and van der Merwe 1996, 2006). This model proposes that physical proximity of MHC and TCR on the surface of an antigen presenting cell and a $\mathrm{T}$ cell, respectively, favors exclusion of bulky transmembrane molecules such as CD45 that play inhibitory roles, in turn permitting TCR signaling. This model relies on several assumptions, most notably that the basal state of $\mathrm{T}$ cells actually represents an active and dynamic equilibrium rather than a static "off" state.

\section{BASAL TCR SIGNALING}

Although signaling events triggered by TCR have been extensively studied, much less is understood about the basal state of $\mathrm{T}$ cells. Active basal or tonic signaling through antigen receptors has been postulated for many years. Klausner and colleagues were the first to show that treatment of unstimulated $\mathrm{T}$ cells with pervanadate, a general PTPase inhibitor, was sufficient to trigger ligand-independent phosphorylation of cellular proteins (Garcia-Morales et al. 1990; Secrist et al. 1993). These data imply that an active equilibrium of kinase and phos- phatase activity must exist even in the absence of ligandmediated receptor stimulation. Further evidence for active signaling in the basal state was provided by the finding that unstimulated, resting thymocytes and peripheral $\mathrm{T}$ cells show high basal $\zeta$ chain ITAM phosphorylation and constitutive association with unphosphorylated ZAP70. This phenomenon requires expression of components of the TCR signaling pathway, including Lck and nonselecting MHC (van Oers et al. 1993, 1994, 1996; Stefanova et al. 2002). Given the presence of basal $\zeta$ phosphorylation in unstimulated $\mathrm{T}$ cells, we speculate that it is actually ZAP-70 phosphorylation and activation, rather than merely $\zeta$ phosphorylation, that serves as the key initiating event in TCR signaling initiation in vivo.

Consistent with these early observations, it has been shown that antigen receptors can indeed signal in a ligand-independent manner in vivo, and this signaling has biological significance. For instance, the pre-TCR signals in an antigen-independent manner during thymic development and promotes TCR- $\beta$ selection (Irving et al. 1998). Expression of the BCR is required for B-cell survival and inducible deletion of the BCR can be rescued with low-level constitutive PI3K activity (Lam et al. 1997; Srinivasan et al. 2009). This provides evidence for a required role for tonic BCR signaling in B-cell survival. However, in contrast to the $\mathrm{BCR}$, inducible deletion of the TCR is associated with relatively long-term Tcell survival, raising the question of which biological function is subserved by putative tonic TCR signaling (Polic et al. 2001). One potential function for basal antigen receptor signaling identified in both $\mathrm{T}$ and $\mathrm{B}$ cells is repression of RAG gene expression (Roose et al. 2003; Srinivasan et al. 2009). It has also been suggested that tonic TCR signaling is important to maintain TCR- $\alpha$ expression (Markegard et al. 2011).

In addition to a direct function in lymphocyte survival and differentiation, it has also been suggested that tonic $\zeta$ phosphorylation and basal signaling in general might serve to sensitize or tune receptors to improve ligand recognition and discrimination (Germain and Stefanova 1999; Stefanova et al. 2002). A related hypothesis is that basal signaling enhances the speed of cellular responses to stimuli (Artomov et al. 2010).

It has been difficult to study basal signaling directly. One approach toward unmasking the basal equilibrium is to perturb either positive or negative regulators of this balance genetically. Although mice harboring constitutively active SFKs (Csk-deficient mice and LckY505F mutant mice) indeed show constitutive TCR signaling that is ligand independent, the resting basal state in such mutant $\mathrm{T}$ cells is quite perturbed, obscuring normal tonic signaling (Imamoto and Soriano 1993; Nada et al. 1993; Pingel et al. 1999; Baker et al. 2000). Optimal strategies for studying the basal state require the use of more subtle, temporally refined perturbations such as hypomorphic alleles and chemical inhibitors. Our laboratory has developed and taken advantage of new experimental tools to subtly perturb and thereby study the basal state. Here we discuss our findings and place our observations in the context of recent work from other laboratories. 


\section{A CHEMICAL-GENETIC STRATEGY FOR RAPID AND SELECTIVE MODULATION OF Csk ACTIVITY}

Csk serves as an essential negative regulator of SFK activity by phosphorylating their carboxy-terminal inhibitory tyrosine (Okada et al. 1991; Bergman et al. 1992; Chow et al. 1993). Constitutive deletion of Csk causes embryonic lethality due to defective neural development that is associated with increased SFK activity (Imamoto and Soriano 1993; Nada et al. 1993). Deletion of Csk in immature thymocytes results in TCR- and MHC-independent development of $\mathrm{T}$ cells with abnormal expression levels of TCRs and coreceptors (Schmedt et al. 1998; Schmedt and Tarakhovsky 2001). This aberrant thymic development is dependent on the presence of Lck and Fyn and suggests that loss of Csk results in the generation of TCR-like signals in the absence of TCR engagement.

Because the SFKs are membrane-anchored but Csk is a cytosolic protein, one way by which Csk activity on SFKs is regulated is its reversible recruitment to the plasma membrane or relocalization to other regions of the cell via an SH2 domain-mediated interaction with phosphorylated transmembrane adaptors such as PAG and LIME (Brdicka et al. 2000; Torgersen et al. 2001; Brdickova et al. 2003; Davidson et al. 2003). However, in contrast to mice deficient in Csk, mice deficient in PAG or LIME or both develop normally, implying the existence of alternative adaptors or recruitment mechanisms (Dobenecker et al. 2005; Xu et al. 2005; Gregoire et al. 2007). Therefore, the spatiotemporal localization of Csk in response to TCR engagement remains poorly understood.

To further investigate how Csk regulates TCR signaling, we generated a novel analog-sensitive allele of Csk, $\mathrm{Csk}^{\mathrm{AS}}$, whose kinase activity can be specifically inhibited by a small molecule (Schoenborn et al. 2011). The Csk ${ }^{\mathrm{AS}}$ allele is designed based on the "analog-sensitive kinase" technology (Liu et al. 1998; Bishop et al. 2000), whereby a conserved, bulky gate keeper residue in the ATP-binding pocket of the catalytic domain is mutated to the smaller glycine residue, resulting in an enlarged pocket that can accommodate a larger analog of the common nonselective kinase inhibitor, PP1. Inhibition of $\mathrm{Csk}^{\mathrm{AS}}$ is highly specific since the analog inhibitor is too large to fit into the ATP-binding pocket of wild-type Csk or other wildtype kinases. To take advantage of this unique allele, we misexpressed membrane-targeted Csk ${ }^{\mathrm{AS}}$ in Jurkat $\mathrm{T}$ cells (Schoenborn et al. 2011), and generated BAC transgenic mice expressing cytosolic $\mathrm{Csk}^{\mathrm{AS}}$ in a Csk-deficient genetic background.

\section{Csk REGULATES T-CELL BASAL STATE, TCR SIGNALING THRESHOLD, AND TCR SIGNAL TERMINATION}

Consistent with prior work (Cloutier et al. 1995), Jurkat $\mathrm{T}$ cells transfected with a membrane-targeted Csk ${ }^{\mathrm{AS}}$ construct showed hyperphosphorylation of Lck at its inhibitory tyrosine (Y505) and were unresponsive to TCR stimulation. However, surprisingly, upon inhibition of membrane-targeted $\mathrm{Csk}^{\mathrm{AS}}$ with its analog inhibitor, instead of simply restoring responsiveness to TCR stimulation, we observed activation of TCR signaling in the absence of TCR ligation. Within seconds of $\mathrm{Csk}^{\mathrm{AS}}$ inhibition alone, Lck phosphorylation at its activating tyrosine (Y394) was markedly increased and was accompanied by strong induction of TCR $-\zeta$ chain phosphorylation as well as the phosphorylation of proximal signaling molecules such as ZAP-70 and LAT. We also observed increased ERK phosphorylation and intracellular calcium, and the up-regulation of CD69 expression. This unexpected ligand-independent TCR-mediated activation clearly underscores the role of Csk in establishing and maintaining the basal signaling state. We propose that expression of membrane-targeted $\mathrm{Csk}^{\mathrm{AS}}$ dampens basal signaling, thus inducing a compensatory rewiring of the TCR signaling network that drives spontaneous TCR signaling following $\mathrm{Csk}^{\mathrm{AS}}$ inhibition. Jurkat signal activation which was induced by simply inhibiting membrane-targeted $\mathrm{Csk}^{\mathrm{AS}}$ was stronger and more sustained compared to that induced by antibody-mediated TCR stimulation, further suggesting that Csk may play a role in TCR signal termination.

\section{Csk INHIBITION ACTIVATES SFKs AND PROXIMAL BUT NOT FULL TCR SIGNALING}

$\mathrm{Csk}^{\mathrm{AS}} \mathrm{BAC} \mathrm{Tg}$ mice, in contrast to our Jurkat misexpression model, express normally regulatable cytosolic Csk rather than membrane-targeted Csk. Importantly, by taking advantage of $\mathrm{Csk}^{+/-}$mice, endogenous Csk could be eliminated by breeding. Viability and T-cell development appear normal in these animals. Upon inhibition of $\mathrm{Csk}^{\mathrm{AS}}$ in primary thymocytes and mature CD4 T cells from these BAC Tg mice, we observed rapid hyperphosphorylation of the activating tyrosines of both Lck and Fyn (Fig. 2). This observation confirms the critical role of Csk in restraining SFK activity in resting primary $\mathrm{T}$ cells to maintain the basal equilibrium and prevent aberrant TCR activation. We anticipated concomitant dephosphorylation of the inhibitory tyrosine of Lck with $\mathrm{Csk}^{\mathrm{AS}}$ inhibition since this tyrosine is a direct Csk substrate. Unexpectedly, in contrast to the activation loop tyrosine, dephosphorylation of the Lck inhibitory tyrosine occurred more slowly and was incomplete, suggesting that pools of Lck may exist that are inaccessible or protected from phosphatases such as CD45.

Importantly, in thymocytes, ZAP-70, LAT, and PLC $\gamma 1$ were phosphorylated comparably to anti-CD3 cross-linking following $\mathrm{Csk}^{\mathrm{AS}}$ inhibition, indicating that even in the absence of segregation of bulky transmembrane phosphatases such as CD45 from the TCR, proximal TCR signaling events can be triggered simply by perturbing Csk activity. Our work implies that Csk relocalization or modulation alone may trigger TCR signal initiation. Unexpectedly, and in contrast to Jurkat T cells, $\mathrm{Csk}^{\mathrm{AS}}$ inhibition was not sufficient to drive efficient downstream ERK phosphorylation or to increase intracellular calcium. This correlated with impaired increases of inositol phos- 


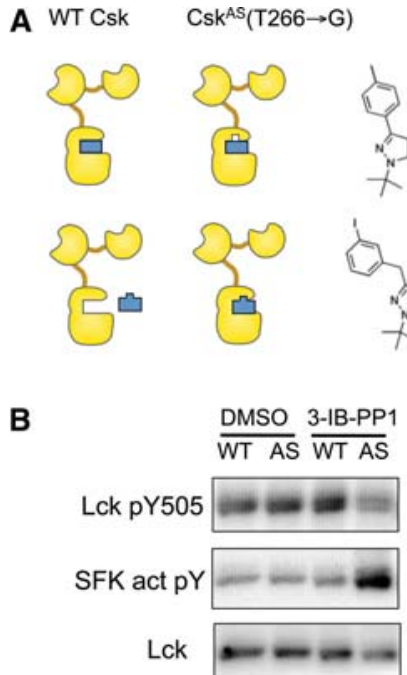

Figure 2. Highly specific inhibition of $\mathrm{Csk}^{\mathrm{AS}}$ allele leads to SFK activation. $(A)$ The bulky gatekeeper residue of wild-type (WT) Csk is mutated from threonine to glycine in the $\mathrm{Csk}^{\mathrm{AS}}$ allele, generating a larger catalytic pocket that can bind a bulkier analog of PP1, 3-IB-PP1. (B) Wild-type (WT) or Csk ${ }^{\mathrm{AS}}$ BAC transgenic (AS) thymocytes were treated with DMSO or $10 \mu \mathrm{M} 3$-IBPP1 for $3 \mathrm{~min}$, and then lysed and analyzed by immunoblotting for SFK phosphorylation using Lck pY505 Ab and Src pY416 $\mathrm{Ab}$ (cell signaling) that recognize the inhibitory phosphotyrosine of Lck and the activation loop phosphotyrosine of the SFKs, respectively (upper band, Fyn; lower band, Lck). Total Lck is probed as a loading control.

phates, suggesting that the defective propagation of TCR signaling to the calcium and MAPK pathways resulted from a failure of PLC $\gamma 1$ to hydrolyze phosphatidylinositol-4,5-bisphosphate $\left(\mathrm{PIP}_{2}\right)$.

The cortical actin cytoskeleton can serve as a barrier for free movement of proteins in or at the plasma membrane (Kusumi et al. 2005). When $\mathrm{Csk}^{\mathrm{AS}}$ was inhibited in BAC Tg thymocytes in the presence of an actin modulating agent such as cytochalasin D, full TCR signaling was restored, and a concomitant increase in intracellular calcium as well as strong ERK phosphorylation was observed. This suggests that the cortical actin cytoskeleton can regulate access of PLC $\gamma 1$ to $\mathrm{PIP}_{2}$. Recently, it was shown that the actin cytoskeleton can restrict lateral diffusion of B-cell transmembrane molecules and limit signaling (Treanor et al. 2010). Alternatively, $\mathrm{PIP}_{2}$ may be sequestered by various actin regulatory proteins and the addition of actin modulating agents may disrupt these interactions and release $\mathrm{PIP}_{2}$ for hydrolysis (McLaughlin and Murray 2005; Di Paolo and De Camilli 2006). Interestingly, inhibiting $\mathrm{Csk}^{\mathrm{AS}}$ in thymocytes in the presence of mature dendritic cells could also restore full TCR signaling. Rescue was dependent on the presence of MHC and B7 molecules on the dendritic cells, suggesting that a major function of TCR and CD28 interaction with these molecules may be to reorganize the actin cytoskeleton.

Although inhibition of $\mathrm{Csk}^{\mathrm{AS}}$ alone in Jurkat cells induced full TCR signaling, this was not the case in Csk ${ }^{\text {AS }}$ BAC Tg T cells where additional stimuli were necessary.
We propose two hypotheses to account for this difference. First, Jurkat $\mathrm{T}$ cells express endogenous Csk and activation of these cells by $\mathrm{Csk}^{\mathrm{AS}}$ inhibition could simply reflect the dominant-negative effects of the $\mathrm{SH} 2$ and $\mathrm{SH} 3$ domains of the transfected membrane-targeted $\mathrm{Csk}^{\mathrm{AS}}$. Second, Jurkat cells have more active basal signaling and stronger inducible signaling due to loss of PTEN and SHIP lipid phosphatases, critical negative regulators of signaling.

Importantly, our work supports a modified version of the kinetic segregation model in which Csk relocalization or modulation may contribute to TCR signal initiation. Indeed, recent work from the Zamoyska laboratory shows a pool of Csk is relocalized to the distal pole in effector CD8 T cells following TCR stimulation (Borger et al. 2013). Surprisingly, we show that activation of SFKs with the $\mathrm{Csk}^{\mathrm{AS}}$ inhibitor is not sufficient on its own to fully trigger TCR signaling and additional actin remodeling may be required for downstream signal propagation. Our studies suggest that the kinetic segregation model is insufficient to account for T-cell activation upon TCR ligation. It has also been demonstrated that costimulation via CD28 mediates actin cytoskeletal changes (Kaga et al. 1998; Tskvitaria-Fuller et al. 2003). Thus, under physiologic signaling conditions where TCR signaling may be limiting, additional requisite actin remodeling could perhaps be provided by CD28 costimulation by its recruitment and activation of $\mathrm{Vav}$, the guanine-nucleotide exchange factor for $\mathrm{Cdc} 42$ and Rac, important mediators of actin polymerization (Salazar-Fontana et al. 2003; Kovacs et al. 2005; Tavano et al. 2006; Boomer and Green 2010).

\section{AN ALLELIC SERIES OF CD45-EXPRESSING MICE}

CD45 phosphatase activity is essential to counteract the function of Csk kinase in regulating Lck carboxyterminal tyrosine phosphorylation. Indeed, CD45-deficient $\mathrm{T}$ cells and cell lines show constitutive hyperphosphorylation of Y505 (Kishihara et al. 1993; Sieh et al. 1993; Byth et al. 1996; Stone et al. 1997; Mee et al. 1999). As a result, CD45 is absolutely required for TCR stimulation and consequently for T-cell development. An Lck Y505F transgene is sufficient to rescue TCR signaling and T-cell development in CD45-deficient mice, establishing this tyrosine as a physiologically relevant substrate and Lck as a critical mediator of CD45 function in vivo (Stone et al. 1997; Pingel et al. 1999; Seavitt et al. 1999).

However, it has been suggested that in addition to its critical positive regulatory role, CD45 also plays a negative regulatory role, and indeed this forms the basis for its putative function in the kinetic segregation model of TCR triggering. Dephosphorylation of the activation loop tyrosine of Lck (Y394) may be the biochemical basis for the negative regulatory role of CD45 (Ashwell and D’Oro 1999; McNeill et al. 2007). Indeed, dual hyperphosphorylation of Lck at both the inhibitory and activat- 
ing tyrosines has been reported in many CD45-deficient cell lines and mice (Ashwell and D'Oro 1999). Several groups have independently generated CD45 single isoform transgenic mice with subphysiologic CD45 expression levels (Kozieradzki et al. 1997; Ogilvy et al. 2003; McNeill et al. 2007). The dual positive and negative regulatory roles of CD45 are unmasked when CD45 expression levels are altered.

To unmask novel functions for CD45, we have taken advantage of an ENU-generated variant of CD45, lightning, with low-surface expression but preserved splicing. By further combining this allele with wild-type and knockout CD45 alleles, we have generated an allelic series of mice in which expression of normally spliced CD45 is varied across a broad range on all hematopoietic cells (Zikherman et al. 2010).

Signaling through the preTCR complex at the thymic TCR- $\beta$-selection checkpoint occurs in a ligand-independent, SFK-dependent manner, and is partially blocked in CD $45^{-/-}$mice (Byth et al. 1996; Irving et al. 1998). Increasing CD45 expression across the allelic series partially rescues this $\beta$-selection defect, but surprisingly, very high CD45 doses were required for complete rescue (Zikherman et al. 2010), as they were for rescue of other basal TCR signaling markers, such as expression of CD5 and TCR- $\beta$ on preselection double positive thymocytes, as well as basal TCR $-\zeta$ chain phosphorylation. Our data suggest that CD45 plays a predominantly positive regulatory role during ligand-independent signaling and that CD45 phosphatase activity must be continuously counter-regulated in the basal state by Csk. To test this hypothesis, we generated CD45 allelic series mice in which the dose of Csk was reduced by half. We observed further partial rescue of these basal signaling phenotypes in allelic series animals with Csk dose reduction. These data were consistent with our model and revealed a tightly regulated and dynamic balance between CD45 and Csk in the basal state.

In contrast to basal signaling, we observed very different sensitivity to CD45 dose in the context of liganddependent TCR signaling. As previously reported (Koretzky et al. 1990, 1991; Stone et al. 1997), CD45 $\mathrm{T}$ cells were refractory to stimulation through the TCR. We observed that even very low levels of CD45 expression could rescue in vitro TCR signaling in response to anti-CD3 ligation. Furthermore, cells with very low CD45 expression were super-sensitive to such TCR ligation. Increasing CD45 expression down-regulated TCR signaling (calcium and phospho-Erk assays) and flattened the dose response curve. This work clearly unmasks a negative role for CD45 in response to in vitro TCR cross-linking. However, by taking advantage of an in vivo reporter of TCR signaling in which GFP expression is under the control of the Nur77 regulatory region, we have recently found that in vivo TCR signaling at the thymic positive selection checkpoint in allelic series mice does not seem to mirror these in vitro assays (Zikherman et al. 2012). This suggests that a positive regulatory role for CD45 may dominate TCR signaling in vivo irrespective of CD45 expression level.

\section{REGULATION OF Lck PHOSPHORYLATION BY CD45}

The effects of different levels of CD45 on TCR signaling, T-cell development and function is most likely a manifestation of different levels of its phosphatase activity. The most well established substrate of CD45 is Lck. We examined the phosphorylation status of the T-cellspecific SFK Lck and confirmed previous observations that phosphorylation of the negative regulatory tyrosine Y505 is highly sensitive to the expression of CD45 (Fig. 3). CD45 dose correlated with the phosphorylation status of Lck Y505. Further, this sensitive titration of Lck Y505 phosphorylation occurred in the basal state and correlated with basal TCR signaling in the CD45 allelic series of mice, suggesting the presence of constitutive counter-regulation of this tyrosine site by Csk.

Interestingly, the activation loop tyrosine of Lck is also sensitive to CD45 dose in resting primary T cells (Fig. 3). Thymocytes with low levels of CD45 have higher levels of phosphorylation at this site, suggesting that CD45 directly or indirectly is capable of dephosphorylating the activation loop tyrosine in addition to the inhibitory tyrosine of Lck, potentially exerting opposing effects on SFK function and activity. However, it should be mentioned that there are other phosphatases, such as PEP, that have also been implicated in the dephosphorylation of Y394 (Cloutier and Veillette 1999). These findings are consistent with results reported in the context of an allelic series of CD45RO transgenic animals (McNeill et al. 2007). We propose that the positive regulatory role of

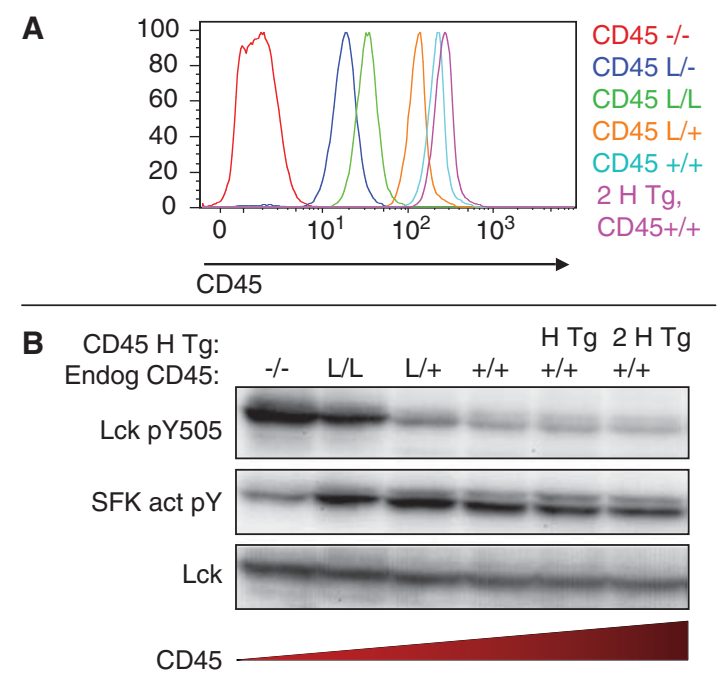

Figure 3. An allelic series of CD45-expressing mice unmasks positive and negative regulation of Lck. $(A)$ Surface CD45 expression assessed by fluorescence-activated cell sorting (FACS) in double-positive thymocytes from CD45 allelic series mice (L, lightning allele; $\mathrm{H} \mathrm{Tg}, \mathrm{CD} 45 \mathrm{H}$ transgene expressed in the context of normal endogenous CD45 expression). (B) Unstimulated thymocytes from allelic series mice are probed for SFK phosphorylation using Lck pY505 Ab and Src pY416 Ab (cell signaling) that recognize the inhibitory phosphotyrosine of Lck and the activation loop phosphotyrosine of the SFKs, respectively (upper band, Fyn; lower band, Lck). Total Lck is probed as a loading control. 
CD45 is directed at the inhibitory SFK tyrosine phosphorylation, whereas the negative regulatory role of CD45 is directed at the activation loop tyrosine of Lck. However, our studies rely upon analysis of whole cell lysates. Discordance between in vitro and in vivo TCR activation in CD45 allelic series $T$ cells raises the possibility that specific pools of Lck are differentially regulated by CD45 and that those pools that are probed via whole cell lysates and in vitro stimulation may not correspond to pools that mediate signaling in vivo.

\section{CD45 AND Csk REGULATE THE BASAL STATE OF T CELLS}

Under basal conditions, Csk is recruited to the plasma membrane by the phosphorylated adaptor PAG (and/or other as yet unidentified adaptors). We propose that dynamic regulation of Csk might account for differential requirements for CD45 during basal and inducible signaling (see the model in Fig. 4). In the basal state, high doses of CD45 are required to dephosphorylate the inhibitory tyrosine of Lck because local concentrations of Csk are high. Under basal conditions therefore, the positive regulatory role of CD45 prevails. Upon antigen receptor ligation, Csk is rapidly removed from the proximity of its substrate by an unclear mechanism, such as, perhaps, rapid dephosphorylation of an adaptor molecule like PAG. Indeed, one must consider alternative adaptors, because the PAG-deficient cells have little phenotypic change (Dobenecker et al. 2005). Moreover recent work suggest that Csk may relocalize to other regions of the cell (Borger et al. 2013). CD45 now acts unopposed upon the inhibitory tyrosine of Lck, such that the dominant factor influencing Lck activity under these circumstances is the phosphorylation status of the activation loop tyrosine. Now the negative regulatory role of CD45 prevails. Indeed, reducing the dose of Csk by half has no effect on in vitro anti-CD3 stimulation of allelic series thymocytes. However, this model fails to account for the discordance between in vitro and in vivo observations of ligand-dependent TCR signaling. These differences may in turn reflect dependence upon different pools of Lck in each of these processes. However, it remains unclear which pools of Lck are most relevant for in vivo ligand-dependent and ligand-independent TCR signaling, and how access of Csk to these pools is regulated dynamically.

\section{SUBCELLULAR POOLS OF Lck}

Work from the Acuto laboratory has investigated the phosphorylation state of different subcellular pools of Lck in $\mathrm{T}$ cells and provides evidence for a basal equilibrium of four subsets of Lck with distinct phosphorylation states and activity levels in resting T cells (Fig. 1) (Nika et al. 2010). One notable feature of this study is the identification of active forms of Lck in unstimulated T cells that does not appear to increase significantly upon TCR stimulation. This raises the possibility that such active Lck pools are relocalized/partitioned relative to the

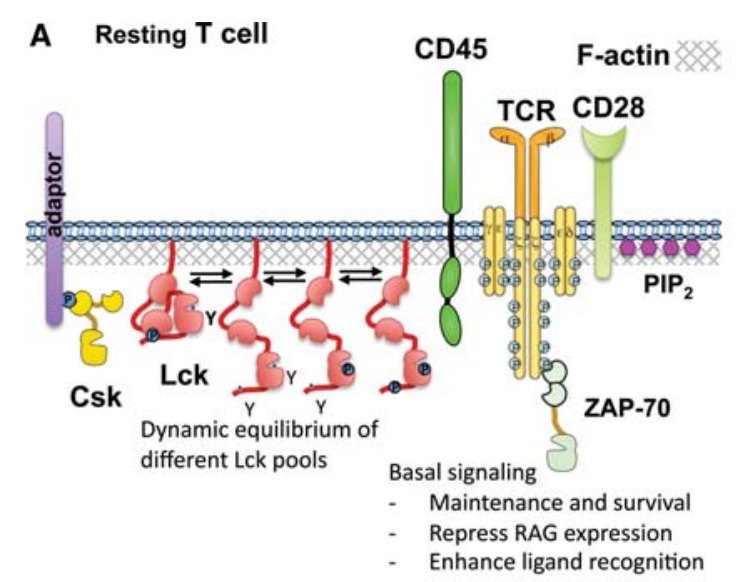

B TCR engaged by agonist pMHC

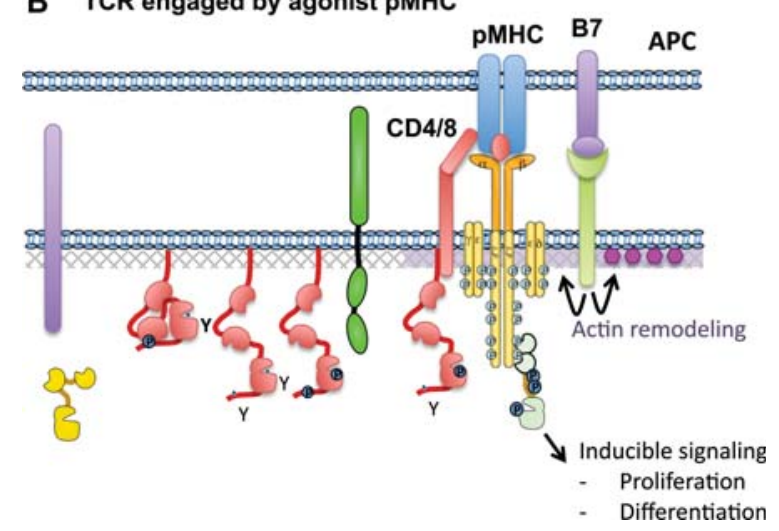

Figure 4. Csk and CD45 regulate T-cell basal and inducible signaling by controlling the dynamic equilibrium of Lck activity. (A) In resting T cells, Csk is recruited to the plasma membrane by membrane-resident adaptors that have yet to be fully identified. Together with CD45, they control the phosphorylation status of the negative regulatory tyrosine of Lck, resulting in the generation of an equilibrium of distinct species of Lck with different levels of activity. It remains to be determined whether each of these Lck species have distinct localizations at the membrane, and whether they differentially associate with CD4/CD8 coreceptors. This equilibrium level of Lck activity sets the T-cell basal signaling tone that may allow for peripheral survival, down-regulation of RAG genes expression as well as increase TCR sensitivity to ligand. $(B)$ When the T cell encounters an antigen presenting cell (APC) bearing its cognate pMHC, the TCR is engaged, inducing the phosphorylation of the TCR ITAMs and ZAP-70 by active Lck. Precisely how this occurs remains poorly defined, and may involve an increase in the amount of active Lck due to a change in the relative activities of Csk and CD45 at the membrane, or a relocalization of preexisting active Lck to the proximity of the TCR. A change in the balance of Csk and CD45 activity may be achieved by relocalization of Csk off the membrane or away from Lck. The additional interaction of CD28 with B7 may initiate actin remodeling events that promote downstream propagation of the proximal TCR signal to the calcium and MAPK pathways, by enabling hydrolysis of $\mathrm{PIP}_{2}$ by PLC $\gamma 1$. Full activation of TCR signaling results in T-cell proliferation and differentiation.

TCR before and after activation of the T cell and that additional activation of Lck may not be important for TCR triggering (Davis and van der Merwe 2011).

However, recent work from the Schraven laboratory with a FRET-based Lck conformational reporter and 
FLIM technology suggests that TCR activation by antiCD3 ligation or SAg correlated with conformational changes in a small pool of Lck with presumed increased Lck kinase activity (Stirnweiss et al. 2013). This has very different implications for the mechanism of TCR triggering. It will be critical to study individual functionally relevant pools of Lck to resolve this controversy, perhaps in the context of $\mathrm{Csk}^{\mathrm{AS}}$ model system and the CD45 allelic series.

\section{CD45, Csk, AND THE KINETIC SEGREGATION MODEL OF TCR TRIGGERING}

CD45 has been postulated to play a critical role in the kinetic segregation model of TCR activation. The Vale laboratory has recently reported reconstitution of heterologous 293 cells with proximal components of the TCR signaling pathway (James and Vale 2012). In this model system, segregation of CD45 away from the TCR-APC interface is both necessary and sufficient for ZAP-70 recruitment to the membrane-associated ITAM domains of the TCR. These data are consistent with the kinetic segregation model of TCR triggering. Recent work from van der Merwe and colleagues also suggests that the large ectodomain of CD45 helps to limit its negative regulatory role during TCR signaling, possibly by limiting association with TCR microclusters (Cordoba et al. 2013).

Our studies of the CD45 allelic series reveal a dual positive and negative regulatory role for this phosphatase, as previously suggested. However, in vivo we find that CD45 has a predominantly positive regulatory role during basal/tonic TCR signaling and apparently during thymic positive selection as well. This feature of CD45 is not evident in the Vale reconstituted cell line model where CD45 has an exclusively negative regulatory role. We speculate that this may be partially due to unconstrained localization of Lck that might miss the important contribution of distinct pools of Lck that are present in primary cells. This model system also focuses exclusively on proximal signaling events such as ZAP-70 recruitment to the membrane and its phosphorylation. Previous studies have shown that ZAP-70 is constitutively associated with phosphorylated TCR $-\zeta$ chains in primary T cells (van Oers et al. 1994). Our recent Csk ${ }^{\text {AS }}$ studies have shown that proximal activation of Lck is actually not sufficient to trigger full T-cell activation. These observations raise the possibility that the reconstituted heterologous cell system may not reflect the normal basal equilibrium of primary $\mathrm{T}$ cells.

The initial proposal of the kinetic segregation model postulated that exclusion of CD45 from the immune synapse was critical in shifting the basal equilibrium and permitting TCR signaling. However, the timescale for CD45 relocalization and the earliest detectable biochemical events upon TCR stimulation seem incommensurate as the latter occur far more quickly than the former. This suggests that CD45 relocalization may help to maintain signaling at the immune synapse but may not account for the initiation of TCR signaling. Our studies of the $\mathrm{Csk}^{\mathrm{AS}}$ model system suggest that modulation of Csk location or activity might contribute to TCR triggering, but suggest that this would also be insufficient. Taken together, this work confirms the contributions of CD45 and Csk to maintain an active basal equilibrium but suggests that acute SFK activation is insufficient to trigger T-cell activation and that some other event is required for full TCR signaling, perhaps involving cytoskeletal rearrangement of the triggered $\mathrm{T}$ cell.

\section{UNANSWERED QUESTIONS}

Our studies and recent work in the field leave a number of important questions unanswered, including the fundamental problem of how TCR ligation leads to T-cell activation. Where is Csk localized relative to its substrate and how is its localization regulated in the basal state and upon TCR triggering? What are the functionally relevant pools of Lck and are these pools activated upon TCR triggering, or merely relocalized? Under which conditions in vivo does CD45 play a negative regulatory role and how can this function be unmasked? Is CD45 enzymatic activity itself regulated or constitutive, and if so, does the extracellular domain contribute via kinetic segregation? Finally, do Csk and CD45 fully account for control of the basal state of T cells? What function does this dynamic equilibrium serve and how is it transformed with TCR triggering? We anticipate that the novel model systems we have developed may serve as experimental platforms from which to address some of these questions.

\section{ACKNOWLEDGMENTS}

This work was supported by the National Institutes of Health/National Institute of Arthritis and Musculoskeletal and Skin Diseases (NIH/NIAMS) (K08 AR059723 to J.Z.), the Arthritis National Research Foundation (to J.Z.), the NIH/National Institute of Allergy and Infections Diseases (NIAID) (PO1 AI091580 to A.W. and Y.X.T.), and the Rosalind Russell Medical Research Foundation Bechtel Award (to J.Z.).

\section{REFERENCES}

Artomov M, Kardar M, Chakraborty AK. 2010. Only signaling modules that discriminate sharply between stimulatory and nonstimulatory inputs require basal signaling for fast cellular responses. J Chem Phys 133: 105101.

Artyomov MN, Lis M, Devadas S, Davis MM, Chakraborty AK. 2010. CD4 and CD8 binding to MHC molecules primarily acts to enhance Lck delivery. Proc Natl Acad Sci 107: 1691616921.

Ashwell JD, D’Oro U. 1999. CD45 and Src-family kinases: And now for something completely different. Immunol Today 20: 412-416.

Baker M, Gamble J, Tooze R, Higgins D, Yang FT, O'Brien PC, Coleman N, Pingel S, Turner M, Alexander DR. 2000. Development of T-leukaemias in CD45 tyrosine phosphatasedeficient mutant lck mice. EMBO J 19: 4644-4654.

Bergman M, Mustelin T, Oetken C, Partanen J, Flint NA, Amrein KE, Autero M, Burn P, Alitalo K. 1992. The human p50csk tyrosine kinase phosphorylates p56lck at Tyr-505 and down regulates its catalytic activity. EMBO J 11: 2919-2924. 
Bishop AC, Ubersax JA, Petsch DT, Matheos DP, Gray NS, Blethrow J, Shimizu E, Tsien JZ, Schultz PG, Rose MD, et al. 2000. A chemical switch for inhibitor-sensitive alleles of any protein kinase. Nature 407: 395-401.

Boniface JJ, Rabinowitz JD, Wulfing C, Hampl J, Reich Z, Altman JD, Kantor RM, Beeson C, McConnell HM, Davis MM. 1998. Initiation of signal transduction through the T cell receptor requires the multivalent engagement of peptide/ MHC ligands [corrected]. Immunity 9: 459-466.

Boomer JS, Green JM. 2010. An enigmatic tail of CD28 signaling. Cold Spring Harb Perspect Biol 2: a002436.

Borger JG, Filby A, Zamoyska R. 2013. Differential polarization of C-terminal Src kinase between naive and antigen-experienced CD8 ${ }^{+}$T cells. J Immunol 190: 3089-3099.

Brdicka T, Pavlistova D, Leo A, Bruyns E, Korinek V, Angelisova P, Scherer J, Shevchenko A, Hilgert I, Cerny J, et al. 2000. Phosphoprotein associated with glycosphingolipid-enriched microdomains (PAG), a novel ubiquitously expressed transmembrane adaptor protein, binds the protein tyrosine kinase csk and is involved in regulation of T cell activation. J Exp Med 191: 1591-1604.

Brdickova N, Brdicka T, Angelisova P, Horvath O, Spicka J, Hilgert I, Paces J, Simeoni L, Kliche S, Merten C, et al. 2003. LIME: A new membrane Raft-associated adaptor protein involved in CD4 and CD8 coreceptor signaling. J Exp Med 198: $1453-1462$.

Byth KF, Conroy LA, Howlett S, Smith AJ, May J, Alexander DR, Holmes N. 1996. CD45-null transgenic mice reveal a positive regulatory role for CD45 in early thymocyte development, in the selection of $\mathrm{CD} 4^{+} \mathrm{CD} 8^{+}$thymocytes, and $\mathrm{B}$ cell maturation. J Exp Med 183: 1707-1718.

Call ME, Wucherpfennig KW. 2004. Molecular mechanisms for the assembly of the T cell receptor-CD3 complex. Mol Immunol 40: $1295-1305$.

Chow LM, Fournel M, Davidson D, Veillette A. 1993. Negative regulation of T-cell receptor signalling by tyrosine protein kinase p50csk. Nature 365: 156-160.

Cloutier JF, Veillette A. 1999. Cooperative inhibition of T-cell antigen receptor signaling by a complex between a kinase and a phosphatase. J Exp Med 189: 111-121.

Cloutier JF, Chow LM, Veillette A. 1995. Requirement of the SH3 and SH2 domains for the inhibitory function of tyrosine protein kinase p50csk in T lymphocytes. Mol Cell Biol 15: 5937-5944.

Cordoba SP, Choudhuri K, Zhang H, Bridge M, Basat AB, Dustin ML, van der Merwe PA. 2013. The large ectodomains of CD45 and CD148 regulate their segregation from and inhibition of ligated T-cell receptor. Blood 121: 4295-4302.

Davidson D, Bakinowski M, Thomas ML, Horejsi V, Veillette A. 2003. Phosphorylation-dependent regulation of T-cell activation by $\mathrm{PAG} / \mathrm{Cbp}$, a lipid raft-associated transmembrane adaptor. Mol Cell Biol 23: 2017-2028.

Davis SJ, van der Merwe PA. 1996. The structure and ligand interactions of CD2: Implications for T-cell function. Immunol Today 17: 177-187.

Davis SJ, van der Merwe PA. 2006. The kinetic-segregation model: TCR triggering and beyond. Nat Immunol 7: $803-$ 809.

Davis SJ, van der Merwe PA. 2011. Lck and the nature of the T cell receptor trigger. Trends Immunol 32: 1-5.

Di Paolo G, De Camilli P. 2006. Phosphoinositides in cell regulation and membrane dynamics. Nature 443: 651-657.

Dobenecker MW, Schmedt C, Okada M, Tarakhovsky A. 2005. The ubiquitously expressed Csk adaptor protein Cbp is dispensable for embryogenesis and T-cell development and function. Mol Cell Biol 25: 10533-10542.

Garcia-Morales P, Minami Y, Luong E, Klausner RD, Samelson LE. 1990. Tyrosine phosphorylation in T cells is regulated by phosphatase activity: Studies with phenylarsine oxide. Proc Natl Acad Sci 87: 9255-9259.

Germain RN, Stefanova I. 1999. The dynamics of T cell receptor signaling: Complex orchestration and the key roles of tempo and cooperation. Annu Rev Immunol 17: 467-522.
Gil D, Schamel WW, Montoya M, Sanchez-Madrid F, Alarcon B. 2002. Recruitment of Nck by CD3 $\epsilon$ reveals a ligand-induced conformational change essential for $\mathrm{T}$ cell receptor signaling and synapse formation. Cell 109: 901-912.

Gregoire C, Simova S, Wang Y, Sansoni A, Richelme S, Schmidt-Giese A, Simeoni L, Angelisova P, Reinhold D, Schraven B, et al. 2007. Deletion of the LIME adaptor protein minimally affects $\mathrm{T}$ and $\mathrm{B}$ cell development and function. Eur J Immunol 37: 3259-3269.

Hermiston ML, Zikherman J, Zhu JW. 2009. CD45, CD148, and Lyp/Pep: Critical phosphatases regulating Src family kinase signaling networks in immune cells. Immunol Rev 228: $288-311$.

Imamoto A, Soriano P. 1993. Disruption of the csk gene, encoding a negative regulator of Src family tyrosine kinases, leads to neural tube defects and embryonic lethality in mice. Cell 73: $1117-1124$.

Irving BA, Alt FW, Killeen N. 1998. Thymocyte development in the absence of pre-T cell receptor extracellular immunoglobulin domains. Science 280: 905-908.

James JR, Vale RD. 2012. Biophysical mechanism of T-cell receptor triggering in a reconstituted system. Nature 487: 64-69.

Kaga S, Ragg S, Rogers KA, Ochi A. 1998. Stimulation of CD28 with B7-2 promotes focal adhesion-like cell contacts where Rho family small $\mathrm{G}$ proteins accumulate in T cells. J Immunol 160: $24-27$.

Kishihara K, Penninger J, Wallace VA, Kundig TM, Kawai K, Wakeham A, Timms E, Pfeffer K, Ohashi PS, Thomas ML, et al. 1993. Normal B lymphocyte development but impaired $\mathrm{T}$ cell maturation in CD45-exon6 protein tyrosine phosphatase-deficient mice. Cell 74: 143-156.

Koretzky GA, Picus J, Thomas ML, Weiss A. 1990. Tyrosine phosphatase CD45 is essential for coupling T-cell antigen receptor to the phosphatidyl inositol pathway. Nature 346: 66-68.

Koretzky GA, Picus J, Schultz T, Weiss A. 1991. Tyrosine phosphatase CD45 is required for T-cell antigen receptor and CD2mediated activation of a protein tyrosine kinase and interleukin 2 production. Proc Natl Acad Sci 88: 2037-2041.

Koretzky GA, Abtahian F, Silverman MA. 2006. SLP76 and SLP65: Complex regulation of signalling in lymphocytes and beyond. Nat Rev Immunol 6: 67-78.

Kovacs B, Parry RV, Ma Z, Fan E, Shivers DK, Freiberg BA, Thomas AK, Rutherford R, Rumbley CA, Riley JL, et al. 2005. Ligation of CD28 by its natural ligand CD86 in the absence of TCR stimulation induces lipid raft polarization in human CD4 T cells. J Immunol 175: 7848-7854.

Kozieradzki I, Kundig T, Kishihara K, Ong CJ, Chiu D, Wallace VA, Kawai K, Timms E, Ionescu J, Ohashi P, et al. 1997. T cell development in mice expressing splice variants of the protein tyrosine phosphatase CD45. J Immunol 158: 3130-3139.

Kusumi A, Nakada C, Ritchie K, Murase K, Suzuki K, Murakoshi H, Kasai RS, Kondo J, Fujiwara T. 2005. Paradigm shift of the plasma membrane concept from the two-dimensional continuum fluid to the partitioned fluid: High-speed singlemolecule tracking of membrane molecules. Annu Rev Biophys Biomol Struct 34: 351-378.

La Gruta NL, Liu H, Dilioglou S, Rhodes M, Wiest DL, Vignali DA. 2004. Architectural changes in the TCR:CD3 complex induced by MHC:peptide ligation. J Immunol 172:3662-3669.

Lam KP, Kühn R, Rajewsky K. 1997. In vivo ablation of surface immunoglobulin on mature B cells by inducible gene targeting results in rapid cell death. Cell 90: 1073-1083.

Levin SE, Weiss A. 2005. Twisting tails exposed: The evidence for TCR conformational change. J Exp Med 201: 489-492.

Liu Y, Shah K, Yang F, Witucki L, Shokat KM. 1998. Engineering Src family protein kinases with unnatural nucleotide specificity. Chem Biol 5: 91-101.

Locksley RM, Reiner SL, Hatam F, Littman DR, Killeen N. 1993. Helper T cells without CD4: Control of leishmaniasis in CD4-deficient mice. Science 261: 1448-1451.

Markegard E, Trager E, Yang CW, Zhang W, Weiss A, Roose JP. 2011. Basal LAT-diacylglycerol-RasGRP1 signals in T cells maintain TCR $\alpha$ gene expression. PLoS One 6: e25540. 
McLaughlin S, Murray D. 2005. Plasma membrane phosphoinositide organization by protein electrostatics. Nature 438: $605-611$

McNeill L, Salmond RJ, Cooper JC, Carret CK, Cassady-Cain RL, Roche-Molina M, Tandon P, Holmes N, Alexander DR. 2007. The differential regulation of Lck kinase phosphorylation sites by CD45 is critical for $\mathrm{T}$ cell receptor signaling responses. Immunity 27: 425-437.

Mee PJ, Turner M, Basson MA, Costello PS, Zamoyska R, Tybulewicz VL. 1999. Greatly reduced efficiency of both positive and negative selection of thymocytes in CD45 tyrosine phosphatase-deficient mice. Eur J Immunol 29: 2923-2933.

Mingueneau M, Sansoni A, Gregoire C, Roncagalli R, Aguado E, Weiss A, Malissen M, Malissen B. 2008. The proline-rich sequence of $\mathrm{CD} 3 \epsilon$ controls $\mathrm{T}$ cell antigen receptor expression on and signaling potency in preselection $\mathrm{CD} 4^{+} \mathrm{CD} 8^{+}$thymocytes. Nat Immunol 9: 522-532.

Nada S, Yagi T, Takeda H, Tokunaga T, Nakagawa H, Ikawa Y, Okada M, Aizawa S. 1993. Constitutive activation of Src family kinases in mouse embryos that lack Csk. Cell 73: $1125-1135$.

Nika K, Soldani C, Salek M, Paster W, Gray A, Etzensperger R, Fugger L, Polzella P, Cerundolo V, Dushek O, et al. 2010. Constitutively active Lck kinase in $\mathrm{T}$ cells drives antigen receptor signal transduction. Immunity 32: 766-777.

Ogilvy S, Louis-Dit-Sully C, Cooper J, Cassady RL, Alexander DR, Holmes N. 2003. Either of the CD45RB and CD45RO isoforms are effective in restoring $\mathrm{T}$ cell, but not $\mathrm{B}$ cell, development and function in CD45-null mice. J Immunol 171: $1792-1800$.

Okada M, Nada S, Yamanashi Y, Yamamoto T, Nakagawa H. 1991. CSK: A protein-tyrosine kinase involved in regulation of src family kinases. $J$ Biol Chem 266: 24249-24252.

Pingel S, Baker M, Turner M, Holmes N, Alexander DR. 1999. The CD45 tyrosine phosphatase regulates CD3-induced signal transduction and $\mathrm{T}$ cell development in recombinase-deficient mice: Restoration of pre-TCR function by active p56(lck). Eur J Immunol 29: 2376-2384.

Polic B, Kunkel D, Scheffold A, Rajewsky K. 2001. How $\alpha \beta$ T cells deal with induced TCR $\alpha$ ablation. Proc Natl Acad Sci 98: $8744-8749$.

Roose JP, Diehn M, Tomlinson MG, Lin J, Alizadeh AA, Botstein D, Brown PO, Weiss A. 2003. T cell receptor-independent basal signaling via Erk and Abl kinases suppresses RAG gene expression. PLoS Biol 1: E53.

Salazar-Fontana LI, Barr V, Samelson LE, Bierer BE. 2003. $\mathrm{CD} 28$ engagement promotes actin polymerization through the activation of the small Rho GTPase Cdc42 in human T cells. J Immunol 171: 2225-2232.

Schmedt C, Tarakhovsky A. 2001. Autonomous maturation of $\alpha / \beta \mathrm{T}$ lineage cells in the absence of $\mathrm{COOH}$-terminal $\mathrm{Src}$ kinase (Csk). J Exp Med 193: 815-826.

Schmedt C, Saijo K, Niidome T, Kuhn R, Aizawa S, Tarakhovsky A. 1998. Csk controls antigen receptor-mediated development and selection of T-lineage cells. Nature 394: 901-904.

Schoenborn JR, Tan YX, Zhang C, Shokat KM, Weiss A. 2011. Feedback circuits monitor and adjust basal Lck-dependent events in T cell receptor signaling. Sci Signal 4: ra59.

Seavitt JR, White LS, Murphy KM, Loh DY, Perlmutter RM, Thomas ML. 1999. Expression of the p56 lck Y505F mutation in CD45-deficient mice rescues thymocyte development. Mol Cell Biol 19: 4200-4208.

Secrist JP, Burns LA, Karnitz L, Koretzky GA, Abraham RT. 1993. Stimulatory effects of the protein tyrosine phosphatase inhibitor, pervanadate, on T-cell activation events. $J$ Biol Chem 268: 5886-5893.

Sieh M, Bolen JB, Weiss A. 1993. CD45 specifically modulates binding of Lck to a phosphopeptide encompassing the negative regulatory tyrosine of Lck. EMBO J 12: 315-321.

Srinivasan L, Sasaki Y, Calado DP, Zhang B, Paik JH, Depinho RA, Kutok JL, Kearney JF, Otipoby KL, Rajewsky K. 2009. PI3 kinase signals BCR-dependent mature B cell survival. Cell 139: 573-586.
Stefanova I, Dorfman JR, Germain RN. 2002. Self-recognition promotes the foreign antigen sensitivity of naive $\mathrm{T}$ lymphocytes. Nature 420: 429-434.

Stirnweiss A, Hartig R, Gieseler S, Lindquist JA, Reichardt P, Philipsen L, Simeoni L, Poltorak M, Merten C, Zuschratter W, et al. 2013. T cell activation results in conformational changes in the Src family kinase Lck to induce its activation. Sci Signal 6: ra13.

Stone JD, Conroy LA, Byth KF, Hederer RA, Howlett S, Takemoto Y, Holmes N, Alexander DR. 1997. Aberrant TCR-mediated signaling in CD45-null thymocytes involves dysfunctional regulation of Lck, Fyn, TCR- $\zeta$, and ZAP-70. J Immunol 158: 5773-5782.

Straus DB, Chan AC, Patai B, Weiss A. 1996. SH2 domain function is essential for the role of the Lck tyrosine kinase in T cell receptor signal transduction. J Biol Chem 271: 9976-9981.

Tavano R, Contento RL, Baranda SJ, Soligo M, Tuosto L, Manes S, Viola A. 2006. CD28 interaction with filamin-A controls lipid raft accumulation at the T-cell immunological synapse. Nat Cell Biol 8: 1270-1276.

Thome M, Duplay P, Guttinger M, Acuto O. 1995. Syk and ZAP-70 mediate recruitment of $\mathrm{p} 56^{\text {lck }} / \mathrm{CD} 4$ to the activated $\mathrm{T}$ cell receptor $/ \mathrm{CD} 3 / \zeta$ complex. J Exp Med 181: 1997-2006.

Torgersen KM, Vang T, Abrahamsen H, Yaqub S, Horejsi V, Schraven B, Rolstad B, Mustelin T, Tasken K. 2001. Release from tonic inhibition of $\mathrm{T}$ cell activation through transient displacement of C-terminal Src kinase (Csk) from lipid rafts. J Biol Chem 276: 29313-29318.

Treanor B, Depoil D, Gonzalez-Granja A, Barral P, Weber M, Dushek O, Bruckbauer A, Batista FD. 2010. The membrane skeleton controls diffusion dynamics and signaling through the B cell receptor. Immunity 32: 187-199.

Tskvitaria-Fuller I, Rozelle AL, Yin HL, Wulfing C. 2003. Regulation of sustained actin dynamics by the TCR and costimulation as a mechanism of receptor localization. J Immunol 171: $2287-2295$.

Van Laethem F, Sarafova SD, Park JH, Tai X, Pobezinsky L, Guinter TI, Adoro S, Adams A, Sharrow SO, Feigenbaum L, et al. 2007. Deletion of CD4 and CD8 coreceptors permits generation of $\alpha \beta T$ cells that recognize antigens independently of the MHC. Immunity 27: 735-750.

van Oers NS, Tao W, Watts JD, Johnson P, Aebersold R, Teh HS. 1993. Constitutive tyrosine phosphorylation of the T-cell receptor (TCR) $\zeta$ subunit: Regulation of TCR-associated protein tyrosine kinase activity by TCR $\zeta$. Mol Cell Biol 13: 5771-5780.

van Oers NS, Killeen N, Weiss A. 1994. ZAP-70 is constitutively associated with tyrosine-phosphorylated TCR $\zeta$ in murine thymocytes and lymph node T cells. Immunity 1: 675-685.

van Oers NS, Killeen N, Weiss A. 1996. Lck regulates the tyrosine phosphorylation of the T cell receptor subunits and ZAP70 in murine thymocytes. J Exp Med 183: 1053-1062.

Wang H, Kadlecek TA, Au-Yeung BB, Goodfellow HE, Hsu LY, Freedman TS, Weiss A. 2010. ZAP-70: An essential kinase in T-cell signaling. Cold Spring Harb Perspect Biol 2: a002279.

Weiss A. 1993. T cell antigen receptor signal transduction: A tale of tails and cytoplasmic protein-tyrosine kinases. Cell 73: 209-212.

Xu H, Littman DR. 1993. A kinase-independent function of Lck in potentiating antigen-specific $\mathrm{T}$ cell activation. Cell 74: $633-643$

Xu S, Huo J, Tan JE, Lam KP. 2005. Cbp deficiency alters Csk localization in lipid rafts but does not affect T-cell development. Mol Cell Biol 25: 8486-8495.

Zikherman J, Jenne C, Watson S, Doan K, Raschke W, Goodnow CC, Weiss A. 2010. CD45-Csk phosphatase-kinase titration uncouples basal and inducible $\mathrm{T}$ cell receptor signaling during thymic development. Immunity 32: 342-354.

Zikherman J, Parameswaran R, Weiss A. 2012. Endogenous antigen tunes the responsiveness of naive $B$ cells but not $T$ cells. Nature 489: 160-164. 


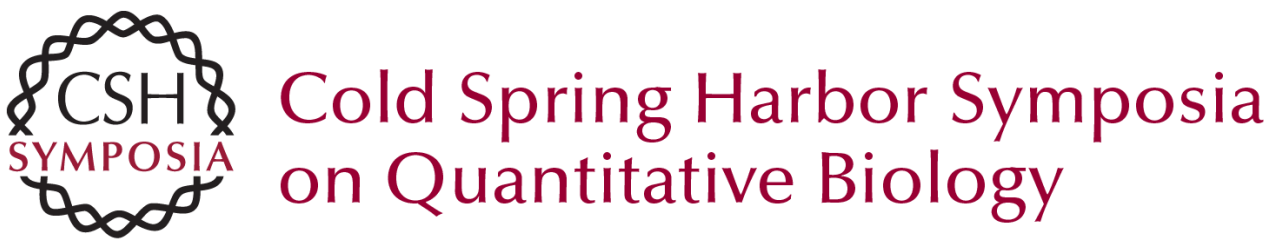

\section{Novel Tools to Dissect the Dynamic Regulation of TCR Signaling by the Kinase Csk and the Phosphatase CD45}

Ying Xim Tan, Julie Zikherman and Arthur Weiss

Cold Spring Harb Symp Quant Biol 2013 78: 131-139 originally published online October 7, 2013 Access the most recent version at doi:10.1101/sqb.2013.78.020347

References This article cites 83 articles, 39 of which can be accessed free at: http://symposium.cshlp.org/content/78/131.full.html\#ref-list-1

License

Email Alerting Receive free email alerts when new articles cite this article - sign up in Service the box at the top right corner of the article or click here. 